\title{
Metadynamics based molecular dynamics simulation of the adsorption of per- and polyfluoroalkyl substances on smectite clay surfaces
}

\author{
JENNIFER WILLEMSEN*, IAN C. BOURG
}

Princeton University, Princeton, NJ 08544, USA

(jarw@princeton.edu,bourg@princeton.edu)

Per- and polyfluoroalkyl substances (PFASs) are a group of persistent, bioaccumulative contaminants that pose an ongoing threat to human health. High levels of PFASs have been detected in water and soils and a fundamental understanding of PFAS adsorption is essential for developing effective remediation strategies and predicting the fate and transport of these contaminants. This research focuses specifically on PFAS interactions with smectite clay, a high surface area mineral readily found in temperate soils.

To understand these interactions, we use molecular dynamics simulations to model a stack of flexible smectite clay sheets with cleaved edges in contact with a bulk aqueous reservoir containing PFAS molecules. Our methodology uses the metadynamics technique to facilitate the exploration of the entire simulation box by the PFAS molecules and to reconstruct the associated free energy landscape. Simulation results are obtained for three PFASs with different fluorinated alkyl chain lengths including perfluorobutanesulfonic acid (PFBS), perfluorohexanesulfonic acid (PFHxS), and perfluorooctanesulfonic acid (PFOS) to examine the effect of PFAS size and hydrophobicity on adsorption. In addition, various aqueous chemistry conditions are examined to study the effect of salinity and coordinating cation type $(\mathrm{K}, \mathrm{Na}, \mathrm{Ca})$ on adsorption.

The simulation output provides atomistic-level insights that help discern mineral-organic interaction mechanisms and conformations (i.e. cation bridging, hydrophobic partitioning), and aid in the interpretation of experimental data. In addition, we calculate the free energy, enthalpy, and entropy of adsorption, the affinity of the PFASs for different clay adsorption sites (interlayer, edge, external basal surface) and examine the effects of clay surface charge density. Combined, these results highlight the importance of entropy as a driving force of PFAS adsorption by smectite clay minerals. 